\title{
Differential attentional bias in generalized anxiety disorder and panic disorder
}

\author{
This article was published in the following Dove Press journal: \\ Neuropsychiatric Disease and Treatment \\ 7 January 2013 \\ Number of times this article has been viewed
}

\section{Jing Chen \\ Zhiyan Wang \\ Yan Wu \\ Yiyun Cai \\ Yifeng Shen \\ Liwei Wang \\ Shenxun Shi}

Department of Psychiatry, Huashan Hospital, Fudan University, Shanghai, People's Republic of China
Correspondence: Shenxun Shi Department of Psychiatry, Huashan Hospital, Fudan

University, 12 Wulumuqi Zhong

Road, Shanghai 200040, People's

Republic of China

Tel +86 2I 52899999

Fax +86 21 62489191

Email shenxun@online.sh.cn
Background: Cognitive theorists relate anxiety disorders to the way in which emotional information is processed. The existing research suggests that patients with anxiety disorders tend to allocate their attention toward threat-related information selectively, and this may differ among different types of anxious subjects. The aim of this study was to explore attentional bias in patients with generalized anxiety disorder (GAD) and panic disorder (PD) using the emotional Stroop task and compare the differences between them.

Methods: Forty-two patients with GAD, 34 patients with PD, and 46 healthy controls performed the emotional Stroop task with four word types, ie, GAD-related words, PD-related words, neutral words, and positive words.

Results: Patients with GAD and those with PD were slower than healthy controls to respond to all stimuli. Patients with GAD had longer response latencies in color-naming both PD-relevant words and GAD relevant words. Patients with PD had longer response latencies only in colornaming PD-related words, similar to healthy controls.

Conclusion: Patients with GAD and those with PD had a different pattern of attentional bias, and there was insufficient evidence to support the existence of specific attentional bias in patients with PD.

Keywords: generalized anxiety disorder, panic disorder, attentional bias, emotional Stroop task

\section{Introduction}

Cognitive theorists relate anxiety disorders to the way in which emotional information is processed. ${ }^{1}$ According to cognitive theories, the attention of patients with anxiety disorders should be biased selectively towards threatening stimuli. The color-word emotional Stroop task has been proposed as an experimental procedure to assess interference between emotion and attention. ${ }^{2,3}$ Longer response latencies when color-naming emotional words reflect attentional bias. A number of studies have administered the emotional Stroop task to patients with anxiety disorders. Some studies have shown that patients with generalized anxiety disorder (GAD) have longer latencies than healthy controls for some types of threat-related words. ${ }^{4,5}$ Although several studies showed an attentional bias for threat information in patients with panic disorder (PD), ${ }^{6-10}$ significant differences when compared with normal controls were found in few of them. ${ }^{6,7}$

The existing research suggests that patients with anxiety disorders tend to allocate their attention selectively toward threat-related information, and that this may differ among subjects with different types of anxiety. Mathews and MacLeod found that patients who worried mostly about physical harm were particularly slow in naming 
the color of physical threat words, whereas patients worrying about social threat were especially slow in naming social threat words. ${ }^{11}$ Other studies found that patients with PD were slowed by physical threat cues, but not social threat cues, whereas the opposite was the case for social phobics. ${ }^{12,13}$ Research conducted in 2001 by Becker et al showed that patients with GAD and those with social phobia demonstrated different types of attentional bias to threat words; the former showed delayed response times for all emotional words, but the latter showed only prolonged reaction times for speechrelated words. ${ }^{4}$

GAD is an anxiety disorder characterized by consistent, uncontrollable, excessive, and often irrational worry. The content of persistent worrisome thoughts may include physical health, work difficulties, study obstacles, or general world problems. PD is an anxiety disorder characterized by recurring severe panic attacks which are periods of intense fear of dying or fear of losing control or going crazy that are of sudden onset. Patients with PD may have persistent concern about having additional attacks and worry about the implications of the attack or its consequences. These two forms of anxiety disorders differ greatly in terms of their manifestations and objects of worry, indicating that patients with GAD and those with PD may have different attentional bias to outside information. Patients with GAD could be expected to have an attentional bias towards a broader range of stimuli than patients with $\mathrm{PD}$, and patients with PD may merely have an attentional bias to negative information related to panic attacks.

No comparative study of differences in attentional bias between GAD and PD have been reported as yet.
In this research, we sought to explore attentional bias in patients with GAD and those with PD using the emotional Stroop task and to compare the differences between them.

\section{Materials and methods Participants}

The study involved three groups of participants, ie, a PD group, a GAD group, and a healthy control group. The PD group consisted of 34 patients who met the Diagnostic and Statistical Manual of Mental Disorders Fourth Edition Text Revision (DSM-IV-TR) ${ }^{14}$ criteria for a diagnosis of PD, comprising five cases of PD with agoraphobia and 29 cases of PD without agoraphobia. Eighteen were medicine-naïve when performing the emotional Stroop task. The GAD group consisted of 42 patients who met the DSM-IV-TR criteria for a diagnosis of GAD. All patients were recruited from the Psychiatry Outpatient Department of Huashan Hospital at Fudan University. The healthy control group consisted of 46 subjects who had never met the diagnostic criteria for any mental disorder listed in the DSM-IV-TR. All healthy controls were relatives of patients treated for carpal tunnel syndrome at the Department of Hand Surgery, Huashan Hospital. Demographic data for all three groups of participants are shown in Table 1. All participants provided their written informed consent before entry to the study.

\section{Materials}

There were four categories of words used in the emotional Stroop task, comprising 24 PD-related words, 24 GADrelated words, 24 neutral words, and 24 positive words.

Table I Demographic information for PD, GAD and HC groups

\begin{tabular}{|c|c|c|c|c|c|}
\hline & $\begin{array}{l}\text { PD } \\
n=34\end{array}$ & $\begin{array}{l}\text { GAD } \\
n=42\end{array}$ & $\begin{array}{l}\text { HC } \\
n=46\end{array}$ & $F / z / \chi^{2}$ & $P$ \\
\hline Gender (M/F) & $11 / 23$ & $19 / 23$ & $|5 / 3|$ & $\chi^{2}=1.920$ & 0.383 \\
\hline Age (year) & $31.59 \pm 8.58$ & $34.33 \pm 7.98$ & $31.07 \pm 7.51$ & $F=2.049$ & 0.133 \\
\hline Marriage (single/married) & $8 / 26$ & $13 / 29$ & $19 / 27$ & $\chi^{2}=2.901$ & 0.234 \\
\hline Education years & $\mid 2.21 \pm 3.43$ & $12.64 \pm 4.36$ & $13.89 \pm 3.21$ & $F=5.102$ & 0.078 \\
\hline Physical laborer & 9 & 9 & 5 & $\chi^{2}=8.265$ & 0.082 \\
\hline White-collar worker & 18 & 30 & 37 & & \\
\hline Unemployed & 7 & 3 & 4 & & \\
\hline Family history (yes/no) & $8 / 26$ & $3 / 39$ & & $\chi^{2}=2.860$ & 0.091 \\
\hline Disease course (month) & $16.97 \pm 18.17$ & $38.78 \pm 37.48$ & & $z=-3.349$ & 0.001 \\
\hline Medicine (naïve/use) & $18 / 16$ & $22 / 20$ & & $\chi^{2}=0.213$ & 0.645 \\
\hline SSRI & 8 & 6 & & & \\
\hline SSRI plus one antianxiety drug & 4 & 6 & & & \\
\hline SSRI plus two antianxiety drugs & 2 & 3 & & & \\
\hline Antianxiety drug & 2 & 5 & & & \\
\hline
\end{tabular}

Notes: Values are the mean \pm standard deviation or numbers; Chi-square tests for categorical variables, analysis of variance for quantitative variables of three groups, and Kruskal-Wallis test for quantitative variables of two groups. Antianxiety drugs included buspirone, trazodone, and benzodiazepines.

Abbreviations: PD, panic disorder; GAD, generalized anxiety disorder; HC, healthy controls; M, male; F, female; SSRI, selective serotonin reuptake inhibitor. 
Those emotional words were selected from the Chinese Affective Words System ${ }^{15}$ and other Chinese studies. ${ }^{16,17}$ The frequency ${ }^{18}$ and strokes of each category of emotional words were matched (see Table 2).

\section{Procedure}

The task procedure was established using E-prime software (PST Inc, Sharpsburg, PA), and the stimulus materials were presented on a 14 inch Lenovo laptop computer. Each stimulus appeared consecutively on the computer screen and participants were instructed to identify the color of the word (red, yellow, green). The size of the words was 50, shown in the center of the computer screen on a white background. As soon as the word appeared on the screen, the participants were required to press one of the three specified buttons on the keyboard corresponding to the color of the word, ignoring the meaning of the word. All words appeared once in one of three possible colors to avoid bias related to color effect or the position of the buttons. A three block design was used, and each stimulus appeared for $1000 \mathrm{msec}$ separated by an interval (white screen) of $500 \mathrm{msec}$. Each block was composed of 96 trials ( 24 words of each word category) pseudo randomized and presented such that neither the same type of word nor the same color could appear consecutively. Before each block, a cross appeared on the screen for 10 seconds in order to enable the participants to get ready. A short break was allowed between each block, and when participants were ready, they pressed the space key to start the next block. Reaction times were recorded between the appearance of the word and the end of the interval $(1500 \mathrm{msec})$. The participants practiced a set of 30 trials before the test session. The stimuli in these trials were color capital letters.

\section{Questionnaires}

The Clinical Interview for DSM-IV-TR Axis I DisordersPatient Edition (SCID-I/P, Chinese edition) was adopted for diagnostic evaluation of the study patients. ${ }^{19}$ Psychiatrists trained in use of the SCID-I/P performed the interview. All participants completed the State-Trait Anxiety Inventory, Self-rating Anxiety Scale, and Self-rating Depression Scale.

\section{Design and data handling}

Pressing a button which did not correspond to the color appearing on the screen was considered to be an error. Colornaming errors and individual reaction times of more than two standard deviations ${ }^{20}$ from a participant's mean and reaction times less than $100 \mathrm{msec}^{5}$ were excluded. We defined the difference between mean reaction times for emotional words and neutral words as the emotional interference score. ${ }^{20}$

Frequency and stroke of the emotional words and participants' mean questionnaire scores were analyzed using the Kruskal-Wallis test and the Wilcoxon rank sun test. A repeated-measures analysis of variance was conducted with the group (three levels, ie, a PD group, a GAD group, and a healthy control group) as a between-subject variable, and word type (four levels, ie, PD-relevant, GAD-relevant, positive and neutral) and block (three levels, blocks 1-3) as within-subject variables. Spearman correlation analysis of emotional interference scores and mean questionnaire scores were conducted. Differences were considered to be statistically significant at a two-sided alpha of 0.05 .

\section{Results}

Mean questionnaire scores for the three groups are shown in Table 3. The numbers of errors for each category of emotional word are presented in Table 4 . There was a significant main effect of word type $\left(F_{3,357}=4.864, P=0.002\right)$, and least squares difference (LSD) post hoc tests showed that the errors on positive words were less than those for the other three types of words (positive words versus neutral words, $P=0.026$; positive words versus GAD-related words $P=0.013$; positive words versus $P D$-related words, $P=0.001)$. No significant main effect of group was observed $\left(F_{2,119}=2.411, P=0.094\right)$, and no interaction between word type and group was observed $\left(F_{6,357}=1.324, P=0.246\right)$.

The reaction times for the three groups of participants are shown in Table 5. There was a significant main effect of word type $\left(F_{3,357}=76.181, P<0.001\right)$ and group $\left(F_{2,119}=7.876\right.$, $P=0.001)$, and the interaction between word type and group was significant $\left(F_{6,357}=2.197, P=0.043\right)$, indicating that participants in the three groups had a different pattern of responses.

Table 2 Frequency and stroke of four types of emotional words

\begin{tabular}{lllll}
\hline & Neutral & GAD-related & PD-related & Positive \\
\hline Frequency & $150.96 \pm 152.61$ & $133.25 \pm 215.40$ & $144.37 \pm 273.16$ & $183.25 \pm 243.88$ \\
Stroke & $18.04 \pm 3.87$ & $18.38 \pm 5.79$ & $19.54 \pm 3.86$ & $18.87 \pm 4.07$ \\
\hline
\end{tabular}

Notes: Values are the mean \pm standard deviation; Kruskal-Wallis test: frequency, $\chi^{2}=6.906, d f=3, P=0.075$; stroke: $\chi^{2}=2.508, d f=3, P=0.474$.

Abbreviations: GAD, generalized anxiety disorder; PD, panic disorder. 
Table 3 Mean questionnaire scores for GAD, PD, and HC groups

\begin{tabular}{|c|c|c|c|c|c|c|c|c|}
\hline & $\begin{array}{l}\text { PD } \\
n=34\end{array}$ & $\begin{array}{l}\text { GAD } \\
n=42\end{array}$ & $\begin{array}{l}\text { HC } \\
n=46\end{array}$ & $\chi^{2}$ & $P$ & PD versus $H C$ & GAD versus HC & PD versus $G A D$ \\
\hline SAI & $48.47 \pm 14.87$ & $44.40 \pm 13.06$ & $28.17 \pm 7.08$ & 47.680 & $<0.001$ & $*$ & $*$ & ns \\
\hline TAl & $50.50 \pm 12.10$ & $49.43 \pm 9.75$ & $31.61 \pm 7.43$ & 59.794 & $<0.001$ & $*$ & $*$ & ns \\
\hline SAS & $57.29 \pm|3.4|$ & $53.00 \pm 9.20$ & $31.65 \pm 5.74$ & 74.544 & $<0.001$ & $*$ & $*$ & ns \\
\hline SDS & $42.85 \pm 9.08$ & $39.17 \pm 8.01$ & $29.02 \pm 6.26$ & 48.566 & $<0.001$ & $*$ & $*$ & ns \\
\hline
\end{tabular}

Notes: Values are the mean \pm standard deviation; Kruskal-Wallis test, use Wilcoxon rank sum test for multiple comparisons; $*$ Wilcoxon rank sum test $P<0.00$; ns, Wilcoxon rank sum test $P>0.05$.

Abbreviations: PD, panic disorder; GAD, generalized anxiety disorder; HC, healthy control; SAI, State Anxiety Inventory; TAI, Trait Anxiety Inventory; SAS, Self-rating Anxiety Scale; SDS, Self-rating Depression Scale.

In the PD group, the reaction times for PD-related words were longer than those for neutral words, and the reaction times for positive words were shorter than those for neutral words $\left(F_{3,99}=35.499, P<0.001\right.$; LSD post hoc tests, PDrelated words versus neutral words, $P<0.001$; positive words versus neutral words, $P<0.001$; GAD-related words versus neutral words, $P=0.447$; GAD-related words versus PDrelated words, $P<0.001$ GAD-related words versus positive words, $P<0.001$; and PD-related words versus neutral words $P<0.001)$. The PD group shared similar variability of reaction times for different word types with that of the control group $\left(F_{3,135}=19.954, P<0.001\right.$; LSD post hoc tests, PD-related words versus neutral words, $P<0.001$; positive words versus neutral words, $P<0.001$; GAD-related words versus neutral words, $P=0.954$; GAD-related words versus PD-related words, $P=0.001$; GAD-related words versus positive words, $P<0.001$; and $\mathrm{PD}$-related words versus positive words, $P<0.001$ ). In the GAD group, the reaction times for GAD-related words and PD-related words were longer than those for neutral words, whereas the reaction times for positive words were shorter than those for neutral words $\left(F_{3,123}=29.741, P<0.001\right.$; LSD post hoc tests, PD-related words versus neutral words, $P<0.001$; positive words versus neutral words, $P<0.001$; GAD-related words versus neutral words, $P<0.001$; PD-related words versus GAD-related words, $P=0.152$; GAD-related words versus positive words, $P<0.001$; and PD-related words versus positive words, $P<0.001)$.

We observed a significant main effect of block $\left(F_{2,238}=14.070, P<0.001\right)$. No interaction between block and group was observed $\left(F_{4,238}=0.315, P=0.868\right)$, indicating that over the three blocks, all three groups of participants were reacting faster, indicating a learning effect, and there was no interaction between block and word type $\left(F_{6,714}=1.595, P=0.146\right)$.

The $\mathrm{PD}$ and GAD groups were divided into a medicine-naïve group and a medicine-experienced group. A repeated-measures analysis of variance was conducted, with group (two levels, ie, medicine-naïve and medicine-experienced) as the betweensubject variable and word type as the within-subject variable. There was no significant main effect of group in patients with GAD and PD (GAD, $F_{1,40}=0.336, P=0.566$; PD, $\left.F_{1,32}=0.108, P=0.744\right)$, nor was there a significant interaction between group and word type in any of the patients (GAD, $F_{3,120}=0.947, P=0.420 ; \mathrm{PD}, F_{3,96}=0.607, P=0.612$ ). There were no significant correlations between emotional interference score and any of the mean questionnaire scores in each of the three groups.

\section{Discussion}

This study shows that, in comparison with controls, participants with GAD and those with PD are slower to respond to all kinds of stimuli, suggesting that attention to an ongoing task may be interfered with in patients with an anxiety disorder. In other words, these patients may have cognitive impairment to some extent. The study conducted by Gualtieri and Morgan showed that substantial numbers of patients with anxiety were cognitively impaired in five domains, ie, memory, psychomotor speed, reaction time, attention, and cognitive flexibility. ${ }^{21}$ Our findings are consistent with their results. However, the strength of our conclusion is limited by the possibility of a false-positive result due to the small sample size.

We found that all three groups of participants had longer response latencies in color-naming PD-relevant words. PDrelevant words such as "impending death", "smothered", and "palpitation", are closely related to human survival. From the perspective of biological evolution, preferential processing of dangerous survival-relevant information tends to be an adaptive process in humans. ${ }^{22}$

We found that patients with GAD had longer response latencies in color-naming both PD-relevant words and GADrelevant words. Becker et al found that patients with GAD took longer than controls to name the color of GAD-related 
Table 4 Error numbers for four types of emotional words in the three groups

\begin{tabular}{llllll}
\hline & Four stimuli & Neutral & GAD-related & PD-related & Positive \\
\hline PD $(n=34)$ & $14.74 \pm 16.13$ & $3.85 \pm 4.26$ & $3.68 \pm 4.35$ & $3.88 \pm 4.58$ & $3.32 \pm 3.98$ \\
GAD $(n=42)$ & $12.05 \pm 9.69$ & $2.74 \pm 2.77$ & $3.29 \pm 2.59$ & $3.64 \pm 3.23$ & $2.36 \pm 2.44$ \\
HC $(n=46)$ & $9.35 \pm 4.81$ & $2.59 \pm 1.92$ & $2.22 \pm 1.53$ & $2.54 \pm 1.91$ & $2.11 \pm 1.61$ \\
\hline
\end{tabular}

Notes: Values are mean \pm standard deviation; repeated-measures analysis of variance: word type effect $F_{3,357}=4.864, P=0.002$; group effect $F_{2,119}=2.41 \mathrm{I}, P=0.094$, interaction between word type and group $F_{6,357}=1.324, P=0.246$.

Abbreviations: PD, panic disorder; GAD, generalized anxiety disorder; $\mathrm{HC}$, healthy controls.

and speech-related words. ${ }^{4}$ Taghavi et al found that children and adolescents with GAD displayed a Stroop interference effect for threat-related as well as depression-related words. ${ }^{5}$ Studies using other experimental procedures, such as visual search tasks and visual probe tasks, also found that patients with GAD had attentional bias to threatening and negative stimuli. ${ }^{23-25}$ Our findings were consistent with the results of these studies. GAD is characterized by excessive worry or concern about a variety of everyday problems, such as health issues, money, family problems, or difficulties at work, even though there is little or nothing to provoke it. The anxiety of the GAD patient is more intense than the situation warrants. The more extensive interference effect observed for patients with GAD might be due to a greater array of stimuli able to trigger the anxiety schema in these patients.

The results of this study show that patients with PD shared a variability of reaction times for emotional words that was similar to that in healthy controls. During the last 20 years, several studies have investigated attentional bias in PD, and inconsistent results have been obtained. The first study in this field was conducted by Ehlers et al in 1988. They found that patients with PD tended to be slower in color-naming threat words, and nonclinical panickers showed greater interference than controls in color-naming threat words. ${ }^{26}$ In a study by Maidenberg et al, patients with PD showed significantly longer response times to PD-related and general threat words than to neutral words. ${ }^{7}$ A study conducted by Hope et al found that panickers had longer latencies for physical threat words. ${ }^{12}$ The results of a study by Lundh et al showed that patients with PD and agoraphobia showed Stroop interference for PD-related words both sub- liminally and supraliminally. ${ }^{6}$ Two recent studies also showed that patients with PD exhibited an attentional bias towards panic-relevant stimuli. ${ }^{27,28}$ Meanwhile, there were some studies which found the opposite results. Some studies found that patients with PD exhibited greater Stroop interference for all threat words, especially those associated with catastrophe ${ }^{8,9}$ and panic-threat words ${ }^{10}$ compared with neutral words, but no significant difference from normal controls was found. Kampman et al did not find differing Stroop interferences between patients with PD and normal controls. ${ }^{29}$ The results of a study by Schneider et al revealed that the children of patients with PD did not show an attentional bias for panic-relevant stimuli. ${ }^{30}$ In a study by De Cort et al, there were no differences in reaction time between a PD group and a healthy control group, or for different word types (panic threat, general threat, and neutral). ${ }^{31}$

Further, we did not find specific attentional bias in patients with PD, and studies had found that other phenomena could suppress a specific Stroop effect. One possible source of suppression is external stressors. ${ }^{32,33}$ In our study, all participants completed the emotional Stroop task under the same conditions. According to the mean questionnaire scores, patients with PD were more anxious than the healthy controls. Nevertheless, correlation analysis revealed no significant relationship between mean State-Trait Anxiety Inventory, Self-rating Anxiety Scale, and emotional interference scores. Differences in mean StateTrait Anxiety Inventory and Self-rating Anxiety Scale scores between the GAD group and the PD group were not significant, whereas they showed different variability of reaction times for emotional words. Therefore, the overall anxiety level during the experiment did not seem to explain the results.

Table 5 Reaction times for four types of emotional words in the three groups

\begin{tabular}{llllll}
\hline & Neutral & GAD-related & PD-related & Positive & Four stimuli \\
\hline PD $(n=34)$ & $656.47 \pm 110.05$ & $654.36 \pm 105.18$ & $671.63 \pm 112.45$ & $641.20 \pm 109.85$ & $655.99 \pm 109.03$ \\
GAD $(n=42)$ & $688.90 \pm 97.79$ & $696.32 \pm 99.85$ & $700.38 \pm 102.29$ & $679.56 \pm 99.32$ & $691.21 \pm 99.26$ \\
HC $(n=46)$ & $614.46 \pm 64.71$ & $614.63 \pm 69.74$ & $625.94 \pm 62.90$ & $602.58 \pm 66.53$ & $614.36 \pm 64.77$ \\
\hline
\end{tabular}

Notes: Values are the mean \pm standard deviation in msec; repeated-measures analysis of variance: word type effect, $F_{3337}=76.18 \mathrm{I}, P<0.00 \mathrm{I} ;$ group effect, $F_{2119}=7.876$, $P=0.00 \mathrm{I}$ (least squares difference post hoc tests, $P D$ versus $H C, P=0.046$; GAD versus $H C, P<0.00 I$; $P D$ versus $G A D, P=0.094$ ), interaction between word type and group $F_{6,357}=2.197, P=0.043$.

Abbreviations: PD, panic disorder; GAD, generalized anxiety disorder; $\mathrm{HC}$, healthy controls. 
Another possible source of suppression of the Stroop effect was the presence of depression..$^{34,35}$ In this study, the mean Self-rating Depression Scale score for the PD group was remarkably higher than that for a control group. No significant relationship between mean Self-rating Depression Scale score and Stroop interference score was observed according to correlation analysis. Further, the difference in mean Self-rating Depression Scale score between the GAD group and the PD group was not significant. Therefore, it was not depression that suppressed the specific Stroop effect in patients with PD.

The subjects were required to respond to 288 stimuli in this research, which was a time-consuming process. One study using a dot-probe task demonstrated that participants with a high level of anxiety showed attentional bias to threatrelated words on the first occasion, and that the attentional bias vanished when the participants were presented with more stimuli. ${ }^{36}$ Although we found a learning effect for the emotional Stroop task, the interaction between block and word type was not significant, indicating that the reaction times for all types of emotional words were becoming faster over the three blocks. Therefore, the lack of specific attentional bias in patients with PD did not result from task habituation.

Patients with PD are characterized by their fear of fear, their bodily sensations, and their catastrophic misinterpretations. Patients with PD may have specific attentional bias towards physical sensations. Palpitation and a sensation of asphyxia are the most common physical symptoms of a panic attack. Kroeze and van Den Hout ${ }^{37,38}$ conducted studies to investigate the attentional bias towards heartbeat information and hyperventilatory sensations in patients with PD. Compared with healthy controls, no evidence was found to support specific selective attention to tachycardia and hyperventilation in patients with $\mathrm{PD}$.

A drawback of the emotional Stroop task is that attentional processes are not measured independently. ${ }^{39}$ The dot-probe detection task does not have this disadvantage. Asmundson and Sandler compared responses to social threat cues, and found that patients with PD had reduced detection latencies to probes presented when they were actively reading stimuli pertaining to a physical threat, and this effect was not observed among control subjects. ${ }^{40}$ However, these investigators failed to replicate their findings in subsequent research, in which no differences were observed in detection latencies for visual probes that followed neutral, social threat, or panic symptom/ fear cues between a PD group and a healthy control group. ${ }^{41}$

To summarize, there is insufficient evidence as yet to support the existence of specific attentional bias in patients with PD. Our study found that errors for positive words were less than those for neutral words, and reaction times for positive words were shorter than those for neutral ones. Previous studies were seldom concerned about words with a positive valence, with some research showing that positive words did not produce a Stroop effect ${ }^{42}$ and that prolonged reaction times were shorter than those for negative words. ${ }^{4}$ In a study by Waters et al, severely anxious children with GAD also showed an attentional bias toward happy faces. ${ }^{25}$ Our results are in contrast with those of Waters et al, possibly because of the varied setting of emotional Stroop tasks. The emotional words were all displayed on cards in early studies, ${ }^{4}$ and the same type of emotional words were presented in succession within a block in the study conducted by Demily et al, ${ }^{42}$ whereas pseudo random presentation was adopted in the present investigation. Research carried out by Zen's study showed that when the emotional words were presented pseudorandomly or presented after positive emotional pictures as effective priming, the reaction times of positive words were shorter than neutral words, ${ }^{43}$ which is consistent with the findings of our study. It is suggested that in circumstances where negative and positive stimuli are presented alternately, the positive stimulus tends to be neglected by subjects, and does not interfere with ongoing cognitive activity.

\section{Limitations}

Several limitations of this study merit discussion. First, its conclusion might be limited due to its small sample size. Second, about half of our patients were on antidepressant medication. The impact of this on the emotional Stroop task in patients with anxiety is not clear. A recent meta-analysis concerning executive dysfunction in unipolar major depressive disorder suggested that significant improvements in Stroop performance might be obtained during the course of antidepressant treatment. ${ }^{44}$ Further, the side effects of other drugs, such as benzodiazepines, might interfere with attentional performance. Therefore, the patients were grouped according to whether they had taken medication or not. Further analysis showed no remarkable difference in performance of the emotional Stroop task between medication users and nonusers, nor was there a correlation between Stroop interference scores and mean questionnaire scores. However, given the small sample size and complex medication used in patients in our study, we cannot fully assess the effect of medication.

For now, a complete system of emotional words like the Affective Norms for English Words has not been devel- 
oped in China. The Chinese Affective Words System only distinguishes positive emotional words from negative ones, but does not further distinguish between different types of negative emotional words. Therefore, when choosing stimulus materials, we referred to other Chinese research.

\section{Conclusion}

Despite the above limitations, the present study is the first to the authors' knowledge to compare differences in attentional bias between patients with GAD and those with PD. We found that patients with GAD and those with PD had different patterns of attentional bias, and that there is not sufficient evidence to support the existence of specific attentional bias in patients with $\mathrm{PD}$ at this time. Future research might include an investigation of the effects of antidepressant medication on Stroop performance.

\section{Acknowledgments}

We would like to thank Yang Zhi, Jiang Yi, Zhou Wen, Zhao Jinping, Institute of Psychology, Chinese Academy of Sciences, and Tang Weijun, Huashan Hospital, Fudan University, Shanghai, for their advice regarding use of the emotional Stroop task.

\section{Disclosure}

The authors report no conflicts of interest in this work.

\section{References}

1. Beck AT, Clark DA. An information processing model of anxiety: automatic and strategic processes. Behav Res Ther. 1997;35(1): 49-58

2. Burt JS. Why do non-color words interfere with color naming? J Exp Psychol Hum Percept Perform. 2002;28(5):1019-1038.

3. Williams JM, Mathews A, MacLeod C. The emotional Stroop task and psychopathology. Psychol Bull. 1996;120(1):3-24.

4. Becker ES, Rinck M, Margraf J, Roth WT. The emotional Stroop effect in anxiety disorders: general emotional or disorder specificity? J Anxiety Disord. 2001;15(3):147-159.

5. Taghavi MR, Dalgleish T, Moradi AR, Neshat-Doost HT, Yule W. Selective processing of negative emotional information in children and adolescents with generalized anxiety disorder. Br J Clin Psychol. 2003;42(Pt 3):221-230.

6. Lundh LG, Wikström J, Westerlund J, Ost LG. Preattentive bias for emotional information in panic disorder with agoraphobia. $J$ Abnorm Psychol. 1999;108(2):222-232.

7. Maidenberg E, Chen E, Craske M, Bohn P, Bystritsky A. Specificity of attentional bias in panic disorder and social phobia. J Anxiety Disord. 1996;10(6):529-541.

8. McNally RJ, Riemann BC, Kim E. Selective processing of threat cues in panic disorder. Behav Res Ther. 1990;28(5):407-412.

9. McNally RJ, Riemann BC, Louro CE, Lukach BM, Kim E. Cognitive processing of emotional information in panic disorder. Behav Res Ther. 1992;30(2):143-149.

10. McNally RJ, Amir N, Louro CE, Lukach BM, Riemann BC, Calamari JE. Cognitive processing of idiographic emotional information in panic disorder. Behav Res Ther. 1994;32(1):119-122.
11. Mathews A, MacLeod C. Selective processing of threat cues in anxiety states. Behav Res Ther. 1985;23(5):563-569.

12. Hope DA, Papee RM, Heimberg RG, Domberk MJ. Representations of the self in social phobia: vulnerability to social threat. Cognit Ther Res. 1990;14(2):177-189.

13. Mogg K, Mathews A, Weinman J. Selective processing of threat cues in anxiety states: a replication. Behav Res Ther. 1989;27(4): 317-323.

14. American Psychiatric Association. Diagnostic and Statistical Manual of Mental Disorders, Fourth Edition, Text Revision. Washington, DC: American Psychiatric Association; 2000.

15. Wang YN, Zhou LM, Luo YJ. The pilot establishment and evaluation of Chinese Affective Words System. Chin Ment Health J. 2008;23(8):608-612.

16. Xu XY, Tao JH. Distinction of emotional category for Chinese emotional system. Presented at the First International Conference on Chinese Affective Computing and Intelligent Interaction. Beijing, China; October 22-24, 2005.

17. Chen SH. Personality and Cognition. Beijing, China: Social Sciences Academic Press; 2005.

18. Liu Y, Liang NY. Words frequency dictionary of modern Chinese. Beijing, China: Astronautic Publishing House; 1990.

19. First MB, Gibbon M, Spitzer RL, Williams JBW. Structured Clinical Interview for DSM-IV-TR Axis I Disorder (Research Version). New York, NY: Biometrics Research, New York State Psychiatric Institute; 2001.

20. Dresler T, Meriau K, Heekeren HR, van der Meer E. Emotional Stroop task: effect of word arousal and subject anxiety on emotional interference. Psychol Res. 2009;73(3):364-371.

21. Gualtieri CT, Morgan DW. The frequency of cognitive impairment in patients with anxiety, depression, and bipolar disorder: an unaccounted source of variance in clinical trials. J Clin Psychiatry. 2008;69(7):1122-1130.

22. Vuilleumier P. Facial expression and selective attention. Curr Opin Psychiatry. 2002;15(3):291-300.

23. Rinck M, Becker ES, Kellermann J, Roth WT. Selective attention in anxiety: distraction and enhancement in visual search. Depress Anxiety. 2003; 18(1):18-28.

24. Mogg K, Millar N, Bradley BP. Biases in eye movements to threatening facial expressions in generalized anxiety disorder and depressive disorder. J Abnorm Psychol. 2000;109(4):695-704.

25. Waters AM, Mogg K, Bradley BP, Pine DS. Attentional bias for emotional faces in children with generalized anxiety disorder. $J$ Am Acad Child Adolesc Psychiatry. 2008;47(4):435-442.

26. Ehlers A, Margraf J, Davies S, Roth WT. Selective processing of threat cues in subjects with panic attacks. Cogn Emot. 1988;2(3): 201-219.

27. Wiener C, Perloe A, Whitton S, Pincus D. Attentional bias in adolescents with panic disorder: changes over an 8-day intensive treatment program. Behav Cogn Psychother. 2012;40(2):193-204.

28. Reinecke A, Cooper M, Favaron E, Massey-Chase R, Harmer C. Attentional bias in untreated panic disorder. Psychiatry Res. 2011; 185(3):387-393.

29. Kampman M, Keijsers GP, Verbraak MJ, Naring G, Hoogduin CA. The emotional Stroop: a comparison of panic disorder patients, obsessivecompulsive patients, and normal controls, in two experiments. JAnxiety Disord. 2002;16(4):425-441.

30. Schneider S, Unnewehr S, In-Albon T, Margraf J. Attention bias in children of patients with panic disorder. Psychopathology. 2008;41(3): 179-186.

31. De Cort K, Hermans D, Spruyt A, Griez E, Schruers K. A specific attentional bias in panic disorder? Depress Anxiety. 2008;25(11):951-955.

32. Constans JI, McCloskey MS, Vasterling JJ, Brailey K, Mathews A. Suppression of attentional bias in PTSD. J Abnorm Psychol. 2004; 113(2):315-323.

33. Mathews A, Sebastian S. Suppression of the emotional Stroop effects by fear-arousal. Cogn Emot. 1993;7(6):517-530. 
34. Grant D, Beck J. Attentional biases in social anxiety and dysphoria: does comorbidity make a difference? J Anxiety Disord. 2005;20(4): 520-529.

35. Musa C, Lepine JP, Clark DM, Mansell W, Ehlers A. Selective attention in social phobia and the moderating effect of a concurrent depressive disorder. Behav Res Ther. 2003;41(9):1043-1054.

36. Liu XH, Qian MY, Zhou XL. Patterns of attentional bias of highly anxious individuals by repeating the occasions of word stimulus. Chin Ment Health J. 2007;21(11):769-772.

37. Kroeze S, van den Hout MA. Selective attention for cardiac information in panic patients. Behav Res Ther. 2000;38(1):63-72.

38. Kroeze S, van Den Hout MA. Selective attention for hyperventilatory sensations in panic disorder. J Anxiety Disord. 2000;14(6):563-581.

39. Algom D, Lev S, Chajut E. A rational look at the emotional Stroop phenomenon: a generic slowdown, not a Stroop effect. J Exp Psychol Gen. 2004;133(3):323-338.
40. Asmundson GJ, Sandler LS. Selective attention toward physical threat in patients with panic disorder. J Anxiety Disord. 1992;6(4):295-303.

41. Asmundson GJ, Stein MB. Dot-probe evaluation of cognitive processing biases in patients with panic disorder: a failure to replicate and extend. Anxiety. 1994;1(3):123-128.

42. Demily C, Attala N, Fouldrin G, et al. The emotional Stroop task: A comparison between schizophrenic subjects and controls. Eur Psychiatry. 2009;25(2):75-79.

43. Zen YF. An Experimental Study of Emotional Stroop Effect in Positive Stimulus. Guangzhou, China: Faculty of Education Sciences, South China Normal University; 2005.

44. Wagner S, Doering B, Helmreich I, Lieb K, Tadic A. A meta-analysis of executive dysfunctions in unipolar major depressive disorder without psychotic symptoms and their changes during antidepressant treatment. Acta Psychiatr Scand. 2012;125(4):281-292.

\section{Publish your work in this journal}

Neuropsychiatric Disease and Treatment is an international, peerreviewed journal of clinical therapeutics and pharmacology focusing on concise rapid reporting of clinical or pre-clinical studies on a range of neuropsychiatric and neurological disorders. This journal is indexed on PubMed Central, the 'PsycINFO' database and CAS.
The manuscript management system is completely online and includes a very quick and fair peer-review system, which is all easy to use. Visit http://www.dovepress.com/testimonials.php to read real quotes from published authors.

Submit your manuscript here: http://www.dovepress.com/neuropsychiatric-disease-and-treatment-journal 